\title{
Reputation based on punishment rather than generosity allows for evolution of cooperation in sizable groups
}

\author{
Miguel dos Santos* and Claus Wedekind \\ Department of Ecology and Evolution, University of Lausanne, 1015 Lausanne, Switzerland \\ *correspondence: miguel.dossantos@unil.ch
}

Citation: dos Santos M., Wedekind C. 2015. Reputation based on punishment rather than generosity allows for evolution of cooperation in sizable groups. Evolution and Human Behavior 36(1): 59-64.

The content of this preprint is identical with the published paper except from small edits made at proofs.

\begin{abstract}
Cooperation among unrelated individuals can arise if decisions to help others can be based on reputation. While working for dyadic interactions, reputation-use in social dilemmas involving many individuals (e.g. public goods games) becomes increasingly difficult as groups become larger and errors more frequent. Reputation is therefore believed to have played a minor role for the evolution of cooperation in collective action dilemmas such as those faced by early humans. Here, we show in computer simulations that a reputation system based on punitive actions can overcome these problems and, compared to reputation system based on generous actions, (i) is more likely to lead to the evolution of cooperation in sizable groups, (ii) more effectively sustains cooperation within larger groups, and (iii) is more robust to errors in reputation assessment. Punishment and punishment reputation could therefore have played crucial roles in the evolution of cooperation within larger groups of humans.
\end{abstract}

\section{Introduction}

Explaining the rather high level of cooperation in humans is still a challenge for economists, social scientists, and evolutionary biologists, despite the variety of mechanisms that are known to promote cooperation (West et al., 2007). These mechanisms can be categorized as conferring indirect fitness benefits (i.e. kin based, if individuals interact more often with related partners; Hamilton, 1964) or direct fitness benefits (if based on, for example, beneficial by-products; Kokko et al., 2001), the latter often being founded on enforcement mechanisms (West et al., 2007). Enforcing mechanisms require conditional behavioural strategies, e.g. punishing defectors or rewarding cooperators. On the one hand, cooperation can be enforced if individuals inflict sanctions on wrongdoers by punishing them (Sigmund, 2007) or by excluding them from the social group in order to avoid any future interactions with them (Guala, 2012; Sasaki \& Uchida, 2013). On the other hand, cooperation can be based on reciprocity if individuals have a tendency to help those who have helped them in the past (i.e. direct reciprocity; Axelrod \& Hamilton, 1981; Sigmund, 2010) or those who have helped others (i.e. indirect reciprocity; Alexander, 1987; Earley, 2010; Nowak \& Sigmund, 
2005; Sigmund, 2012). In the latter case, an individual's behaviour needs to be translated into a reputation by a set of rules that must be largely adopted within a social group.

One simple rule that has been used in previous analyses is that individuals earn a good reputation (good image score) by being cooperative, and a bad reputation (bad image score) when defecting (Nowak \& Sigmund, 1998). It has been shown that image scoring could sustain cooperation even in the presence of more selfish strategies (e.g. see Leimar \& Hammerstein, 2001) if reputation is not only binary (either "good" or "bad") but comprises a third state "neutral" (Tanabe et al., 2013). Such a straightforward reputational mechanism enhances cooperation frequency and allows cooperative members of a group to recognise and trust each other (Nowak \& Sigmund, 1998; Wedekind \& Braithwaite, 2002; Wedekind \& Milinski, 2000; Yoeli et al., 2013) and to benefit from choosing their partners (Fu et al., 2008; Sylwester \& Roberts, 2010), creating a biological market based on cooperativeness, i.e. competition among potential partners to be chosen for social interactions (Barclay, 2013).

Many conditional behavioural strategies are known to promote cooperation in dyadic interactions, but cooperation can easily break down in collective action problems, i.e. when more than two individuals are involved (Hardin, 1968). Cooperation frequency in both, direct and indirect reciprocity, is predicted to decline rapidly with increasing group size (Boyd \& Richerson, 1988; Fehr, 2004; Suzuki \& Akiyama, 2005, 2007). Implementing positive incentives (rewards to co-operators) can stimulate cooperation in large groups but fails to stabilize it (Hauert, 2010; Sigmund et al., 2001). Moreover, as group size grows, the probability of knowing everybody's reputation in a group decreases and errors become more likely. The role of reputation-based cooperation in collective action dilemmas has therefore frequently been questioned (Fehr, 2004; Suzuki \& Akiyama, 2005), and probably requires to be linked with reputation from other types of interactions (e.g. alternate dyadic interactions). Nevertheless, humans often show high levels of cooperation in collective action dilemmas.

Here, we test whether a reputation mechanism based on punitive actions can support the evolution of cooperation in groups of unrelated individuals. Punishment can increase cooperation levels if there are opportunities to punish defectors, that is, decreasing a defector's immediate payoff at a personal immediate cost (Fehr \& Gächter, 2002; Raihani et al., 2010; Raihani et al., 2012; Sigmund, 2007). Until recently, such costly punishment has been perceived as an evolutionary puzzle because punishers accept costs to harm others while third parties benefit from the increased cooperation levels (Dreber et al., 2008; Fehr \& Gächter, 2002; Rankin et al., 2009; Sigmund, 2007; Wu et al., 2009). However, if punishers can build up a punishment reputation that affects later decisions of others, punishment can lead to long-term benefits that compensate or even outweigh the immediate costs of punishment (dos Santos et al., 2011; dos Santos et al., 2013; Hilbe \& Sigmund, 2010; Hilbe \& Traulsen, 2012). This holds for dyadic interactions, but it is unclear whether punishment reputation facilitates the emergence of cooperation also groups larger than 2 . We therefore compare the evolution of two types of reputational mechanisms in the same public goods game (PGG) followed by punishment, namely a reputation of being generous and a punishment reputation. In a separate third scenario, we consider a PGG followed by a rewarding stage and reputation based on rewards.

Our analyses confirm that a reputational mechanism based on generosity is unlikely to lead to cooperation in larger groups because such mechanisms are sensitive to memory constrains, to errors in reputation assessment, and even to low frequencies of defection. Adding reward to cooperators allowed for the emergence of cooperation but turned out to be 
unstable since a population of rewarders can be quickly invaded by non-rewarders, as predicted from previous analyses (Hauert, 2010; Sigmund et al., 2001). We find that a reputational mechanism based on punitive actions largely solves these problems, i.e. it resists higher error rates in reputation assessment and frequently allows for the evolution of stable cooperation within larger groups.

\section{Model}

We model a population of finite size $N$, with individuals playing on average $m$ interactions per generation over which they can build up a reputation. For each interaction, one group of size $n$ is randomly formed while the rest of the population observes the interaction. The total number of interactions per generation is fixed and equal to $m N / n$, hence individuals play $m$ interactions on average. Since strategies cannot condition their behaviour on the number of interactions, no end game effects are possible. An interaction always comprises two stages: a public goods game followed by a punishment stage. In the first stage, all group members play a public goods game where they can contribute or not to a public good by investing an amount $c$. Their decision to contribute is based on their coplayers' reputation (see below). The sum of all contributions is multiplied by a factor $r$ (with $1<r<n$ ) and then equally divided among all group members. As in previous models (Suzuki \& Akiyama, 2005), we exclusively investigated cases where $r$ is a function of group size such that the incentive to cooperate remains similar as group size grows. In the second stage, individuals have the opportunity to impose fines on each defector within their group by paying an amount $\alpha$ for the defector to lose $\beta$, with $\alpha<\beta$. The punishment decision is binary such that deciding to punish implies punishing all defectors irrespective of their number, and punishers have to pay $\alpha$ for each defector. At the end of a generation, individuals from the current generation are selected with replacement in proportion to their payoff to be the parent of a new offspring (the absolute value of the minimum possible payoff +1 is added to all individuals in order to avoid negative or zero payoffs). This process is repeated until the offspring population reaches $N$. A parent transmits culturally its strategy ( $\{x, y, z\}$; see below) to its offspring (analogous to genetic transmission; Cavalli-Sforza, 1981). Transmission errors (mutations) occur at rate $\mu$ and lead to the replacement of an offspring's strategy at random by another one. Hence all strategies (i.e. all combinations of $x, y, z$ ) have an equal probability of arising through mutation.

We first investigate two reputation mechanisms in this public goods game followed by punishment. The only difference between these two conditions is the information used by the players, which allows us to isolate the effect of information. In one case, reputation is based only on cooperative and non-cooperative actions (i.e. generosity scoring). In the other case, reputation is only based on punitive and non-punitive actions (i.e. punishment scoring). Under both reputation systems, an individual's reputation can only be in one of 3 different states: $\{-$ $1,0,+1\}$. Under generosity scoring, only cooperative and uncooperative actions during the PGG stage of an interaction can affect reputation: an individual's reputation, starting at 0 , shifts to +1 after a contribution to the public good and shifts to -1 after a defection. For example, the reputation of a defector $(-1)$ will directly switch to +1 if she cooperates. Therefore, only the previous interaction influences a player's reputation (no difference in cooperation rate was found with a system under which reputation would first switch to 0 and potentially later to +1 in the case of a cooperative act, or from +1 to 0 and then -1 in the case of two consecutive defections). Under punishment scoring, only punitive and non-punitive actions during the punishment stage of an interaction can affect reputation. Here, an individual's reputation starts at 0 , shifts to +1 after punishing defectors, and shifts to -1 after not punishing defectors. 
We further investigate a third reputation system based on rewards (i.e. reward scoring). In this condition, the punishment stage is replaced by a rewarding stage: individuals can reward those who contributed to the public good by paying a cost $\alpha$ for the other to get $\beta$. Here, reputation is only based on whether an individual has rewarded or not contributors to the PGG: an individual's reputation starts at 0 , shifts to +1 after rewarding cooperators, and shifts to -1 after not rewarding cooperators.

Each interaction is public and everybody knows everybody's reputation, i.e. who contributed to the public good (under generosity scoring) or punished defectors (under punishment scoring) in their last interaction. We implemented errors (e.g. based on memory constrains) in reputation assessment. In each interaction, a focal individual has a probability $\varepsilon$ for each of her $n-1$ group members of forgetting their reputation. If this happens, the focal individual acts as if their reputation was 0 . Consequently, the probability that a focal individual knows the reputation of all her $n$-1 group members is $(1-\varepsilon)^{n-1}$.

Individuals are defined by 3 traits: $x, y$ and $z$. The first two, $x$ and $y$, determine an individual's behaviour in the public good game according to the following action rule: contribute an amount $c$ to the public good if there are in the group at least $x \in\{1,2, \ldots, n-1\}$ players with reputation equal to $y \in\{-1,0,+1\}$. For example, an individual with $x=5$ and $y=$ +1 will contribute to the public good if there are at least 5 other group members with reputation +1 . For the punishment stage, the trait $z \in\{0,1\}$ determines an individual's punishment strategy (i.e. never punish/punish defectors). We also added unconditional defectors (ALLD) and cooperators (ALLC) which could both either not punish (i.e. ALLD-0; ALLC-0) or punish (i.e. ALLD-1; ALLC-1). These strategies were added in this way and not simply by letting $x$ range from 0 to $n$, because the latter option would have created a surplus of unconditional strategies (i.e. individuals with $x=0$ but with different $y$ would all be ALLC, similarly individuals with $x=n$ but with different $y$ would all be ALLD).

All simulation runs started with a population of ALLD-0. If not stated otherwise, we use $N=500, m=5, c=1, \mu=0.002, \varepsilon=0$, and 20,000 generations in all our simulations. Table 1 lists all symbols used in the model. Average cooperation frequencies are calculated across 10 replicates for generations 15,000 to 20,000 .

We have performed additional simulations with larger population sizes $(N=2500$, $5000,10000)$ subdivided into $(25,50,100$, respectively) social groups of 100 individuals in order to reduce the effect of drift. Indeed, drift could have been an important evolutionary force in our simulations compared to selection because the population was composed by one single social group (Leimar \& Hammerstein, 2001). Since the probability of a neutral mutant going to fixation through drift in a finite population is $1 / N$, increasing population size allows us to reduce this probability. We reduced mutations rates accordingly. In order to avoid kin selection in this socially structured population, we use panmictic reproduction where the offspring of a parent in a given social group has an equal probability of ending up in any social group, including the parent's. We modelled this process by random formation of social groups at the beginning of each generation. This amounts to a migration rate for an offspring of $1-1 / g$, where $g$ is the number of social groups. Results for our larger subdivided populations with reduced drift effects confirmed our results with higher levels of cooperation and robustness to errors under punishment scoring than generosity scoring (Fig. A12-15).

\section{Results}


In general, increasing group size decreased cooperation frequency, but higher levels of cooperation were reached when decisions were based on reputation from punitive instead of cooperative actions (Fig. 1; Fig. A1). Cooperation emerged rarely under generosity scoring because the evolutionary process usually led to unconditional defectors (ALLD-0) being invaded by discriminatory strategies (i.e. with $0<x<n$ ) that do not differ phenotypically from defectors as they only cooperate if they find themselves by chance in a group with sufficient cooperators (Fig. A2). Even if sufficient numbers of cooperators would be present, any less tolerant strategy (i.e. with a greater $x$ value) would perform better as they would reap the benefit of the public good without contributing. However, when cooperation emerged with generosity scoring, periods of cooperative states could be stabilized with punishment (Fig. A3). Cooperation frequently emerged under reward scoring. However, a population of rewarders is likely to be invaded by non-rewarders, i.e. cooperation turned out to be unstable under these conditions (Fig. A4 and A5).

Under punishment scoring, the dynamics during the first few thousand generations were similar to those under generosity scoring, i.e. discriminatory non-punishing strategies could invade ALLD-0 (Fig. A6). The crucial difference to generosity scoring was that as soon as a few punishers appeared in the population, individuals with a more tolerant strategy (i.e. with a lower $x$ value) not only avoided punishment more often but also contributed to the public good and therefore increased the punishers' fitness. Hence, punishers were frequently able to invade populations of discriminatory individuals and establish cooperation (Fig. A6). However, periods of cooperation could collapse when discriminatory strategies were invaded by punishing unconditional cooperators (i.e. ALLC-1). The latter can be invaded by nonpunishing unconditional cooperators (ALLC-0), who can in turn be invaded by ALLD-0 (Fig. A7). Fig. A8 and A9 show that cooperation under punishment scoring could also emerge more easily for different punishment ratios.

Cooperation in a reputation system based on punitive actions was more robust to higher probabilities of forgetting the others' reputation (Fig. 2; Fig. A10). In most cases, the most tolerant discriminatory strategy $\{x=1, y=1, z=0\}$ invaded ALLD- 0 . This paved the way for punishing discriminators $\{x=1, y=1, z=1\}$ who brought cooperation and stabilized it with punishment of non-contributors (Fig. A11).

\section{Discussion}

Cooperation through a reputation-based mechanism was previously believed to be problematic for social dilemmas involving more than two individuals (Fehr, 2004; Suzuki \& Akiyama, 2005) because increasing group size made it harder to keep track of everybody's reputation while cooperation easily breaks down after a few defections or errors. Our results confirm this view for reputation mechanisms that score generosity. Cooperating rarely evolved if reputation was based on generosity, and if it evolved, group size and the probability of errors in memory or information transfer were both low. A reputation system based on rewards does not fare any better. While favouring the emergence of cooperation, it fails to stabilize it since rewarders can easily be invaded by second-order free-riders who avoid the cost of rewarding, as found previously (Hauert, 2010; Sigmund et al., 2001).

However, if punishers can build up a punishment reputation, cooperation can evolve even in public goods games played by large numbers of players. We found that such a reputational mechanism is tolerant to errors in assessments, because cooperation is likely if 
few punishers in a group are recognized by their punishment reputation. In fact, if a player knows that there is at least one punisher in her group, switching from defection to cooperation increases her payoff by $\beta-c(1-r / n)$, i.e. the gain from not being punished (provided $\beta>c(1$ $-r / n)$ ) reduced by the net share from the PGG. In the case of reputation-based generosity, if a player knows that there is at least one contributor in the group, switching from defection to cooperation decreases her payoff by $c(1-r / n)$. Therefore, as discriminators are better off detecting punishers instead of contributors, punishers will induce more contributions and will then be able to recoup the costs of punishing more rapidly.

So far, the evolution of cooperation and punishment through reputation was mainly investigated in dyadic interactions (dos Santos et al., 2011; Hilbe \& Sigmund, 2010; Hilbe \& Traulsen, 2012). A few studies considered the effects of punishment in larger group sizes but with strategies that were not directly based on individual punishment reputation, or the effect of errors in reputation assessment was not investigated (Boyd \& Richerson, 1992; Brandt et al., 2003; Gardner \& West, 2004). Boyd \& Richerson (1992) showed that in an n-person Prisoner's Dilemma, cooperation could be increased if individuals interacted repeatedly with the same group members and if they could use a strategy that never contributes until punished once. Brandt et al. (2003) studied the effects of information about punitive actions in groups of three in a spatial public goods game. They showed that cooperation was promoted when individuals knew whether their two neighbours were punishers or not. Gardner and West (2004) found that cooperation was favoured if individuals adjust their cooperation level to the average punishment present in the population. It may appear that the cooperative strategies used in these two studies required less demanding cognitive abilities than strategies based on individual reputation. However, they would be vulnerable to the invasion of more discriminatory strategies that could recognize individual punishers and defect in their absence.

Our reputation system based on contributions to the PGG (i.e. generosity scoring) was similar to classical image scoring (Nowak \& Sigmund, 1998) but with a trinary reputation (as in Tanabe et al., 2013) in the sense that no second-order information was taken into account. Therefore, a focal player who does not contribute to the PGG in a group of cooperators or in a group of defectors would be perceived similarly badly. It therefore remains an open question whether strategies using second-order information in generosity scoring (e.g. 'standing'; Leimar \& Hammerstein, 2001) would sustain cooperation in large groups or which assessment norm could possibly evolve (e.g. conferring a bad reputation to individuals defecting when all other group members are defectors, or conferring a bad reputation to individuals defecting when only half of other group members are defectors, and so on...). We believe such system would not be viable since a focal observer would need to correctly know the reputation of all the actor's coplayers to assess the actor's reputation, which is unlikely in the presence of errors and becomes increasingly unlikely as group size grows. Merely monitoring the presence of a few punishers seems both less subject to errors and less cognitively demanding.

We have restrained our model to randomly formed groups, that is, we did not allow for partner choice or population structure for example. Hence assortment between cooperators could not arise. Reputation-based partner choice has been shown to promote cooperation in dyadic interactions (Fu et al., 2008; Sylwester \& Roberts, 2010). An empirical study by Rockenbach \& Milinski (2011) has looked at the effect of partner choice for future interactions in an n-person Prisoner's Dilemma. They found that about half of the subjects actively avoided low contributors but did not take into account punishment decisions for their choice, while the other half chose randomly. However, greater levels of cooperation were 
found when punishment was present, and punishers almost never (i.e. $<3 \%$ of the cases) tried to hide their presence when given the opportunity, suggesting that they wanted others to know that they would punish low contributors. Therefore, our model restriction seems conservative with regard to the evolution of cooperation, i.e. partner choice is likely to further enhance cooperation frequency in a public game with punishment reputation.

It has been argued that a good reputation could not only allow for partner choice in cooperative interactions or receiving more help in future interactions (Alexander, 1987; Roberts, 1998), but also that it might be linked to the actor's quality (Zahavi, 1977), serving as an honest signal conveying information on the actor's resources, capabilities or reproductive quality for example (Barclay, 2013; Fehrler \& Przepiorka, 2013; Tognetti et al., 2014). Consequently, costs of generosity in collective action dilemmas might be outweighed in other various contexts. In contrast to our model where all individuals have similar abilities to contribute or punish, models linking helping behaviors and signals require the more realistic assumption that individuals differ in their condition, e.g. good vs low quality individuals (Gintis et al., 2001; Lotem et al., 2002; Barclay \& Reeve, 2012). In such conditions, it becomes beneficial to monitor one's group members' quality and/or helping behaviours. However, the problem of monitoring everybody correctly remains and will grow difficult with increasing group size. In addition, it is plausible to assume that punitive behaviors also convey similar and/or different information on the punishers' condition. Whether monitoring the others' punitive or cooperative behaviours will be more beneficial from an individual's point of view and whether it will better enhance cooperation will depend on the relative information conveyed by both behaviors and their advantage in other contexts. Hence, linking not only helping but also punitive behaviors to quality in different social contexts remains an interesting line of research.

A previous model by Panchanathan \& Boyd (2004), inspired by empirical work (Milinski et al., 2002), has shown that alternating stages of PGG with dyadic "mutual aid" interactions and linking reputation from both stages could maintain the population in a cooperative state. Contributors to the PGG gain a good reputation and are later more likely to receive help in dyadic interactions. With such mechanism, cooperators are able to socially exclude non-contributors to the PGG at no personal cost, whereas in our model punishing free-riders was individually costly. This mechanism seems able to stabilize cooperation more efficiently than ours. However it does not provide a solution to the evolution of cooperation in collective actions because strategies that link reputation from both stages are unable to invade strategies that do not, leaving open the question of how collective action arises (Panchanathan $\&$ Boyd, 2004). In contrast, our model has shown the initial emergence of cooperation and punishment from rare. Furthermore, in order to effectively direct help towards contributors to the PGG during the "dyadic interaction" stage, individuals again need to monitor correctly who contributed or not in the previous PGG interaction. It remains unknown whether observing punitive behaviors under these circumstances would confer similar advantages as the in our model.

A further conservative assumption of our model (with respect to the emergence of cooperation) is that punishment could not be conditional to the number of punishers in the group (Boyd et al., 2010). However, because only one or a few individuals need to punish defectors in order to maintain high levels of cooperation within a group, punishment in nplayer public goods games can also be studied as a volunteer's dilemma (Przepiorka \& Diekmann, 2013; Raihani \& Bshary, 2011). The benefit of punishment is then a non-linear function of the number of punishers, i.e. punishment could become more effective if used 
conditionally. However, such conditional strategies lead to new dilemmas, and there is a need to explore the effects of punishment and punishment reputation implemented as a volunteer's dilemma in n-players games.

Our study further highlights the potential significance of punishment reputation for the evolution of cooperation not only in dyadic interactions but also in public goods games, i.e. cooperation dilemmas faced simultaneously by larger groups. Allowing reputation to be based on punitive rather than cooperative actions leads to higher levels of cooperation in larger groups and is less affected by memory constrains. We conclude that the interplay between punishment and punishment reputation must have played a crucial role for the evolution of social behaviours within early human groups facing collective action dilemmas.

\section{Acknowledgements}

We thank F. Adler, R. Bshary, T. Day, A. Gardner, D. Hruschka, L. Lehmann, S. Powers, L. Sundström, and two reviewers for discussion and comments on the manuscript.

\section{References}

Alexander, R. D. (1987). The biology of moral systems. Aldine de Gruyter, New York, NY. Axelrod, R., \& W. D. Hamilton. (1981). The evolution of cooperation. Science, 211, 13901396. (DOI 10.1126/science.7466396)

Barclay, P. (2013). Strategies for cooperation in biological markets, especially for humans. Evolution and Human Behaviour, 34,164-175. (DOI 10.1016/j.evolhumbehav.2013.02.002)

Barclay, P., \& H. K. Reeve. (2012). The varying relationship between helping and individual quality. Behavioral Ecology, 23, 693-698. (DOI 10.1093/beheco/ars039)

Boyd, R., H. Gintis, and S. Bowles. (2010). Coordinated punishment of defectors sustains cooperation and can proliferate when rare. Science, 328, 617-620. (DOI 10.1126/science.1183665)

Boyd, R., \& P. J. Richerson. (1988). The evolution of reciprocity in sizable groups. Journal of theoretical Biology, 132, 337-356. (DOI 10.1016/S0022-5193(88)80219-4)

Boyd, R., \& P. J. Richerson. (1992). Punishment allows the evolution of cooperation (or anything else) in sizable groups. Ethology and Sociobiology,13, 171-195. (DOI 10.1016/0162-3095(92)90032-Y)

Brandt, H., C. Hauert, and K. Sigmund. (2003). Punishment and reputation in spatial public goods games. Proceedings of the Royal Society B-Biological Sciences, 270, 1099-1104. (DOI 10.1098/rspb.2003.2336)

Cavalli-Sforza, L. L. L. (1981). Cultural transmission and evolution: a quantitative approach. Princeton University Press.

dos Santos, M., D. J. Rankin, and C. Wedekind. (2011). The evolution of punishment through reputation. Proceedings of the Royal Society B-Biological Sciences, 278, 371-377. (DOI 10.1098/rspb.2010.1275)

dos Santos, M., D. J. Rankin, and C. Wedekind. (2013). Human cooperation based on punishment reputation. Evolution, 67, 2446-2450. (DOI 10.1111/evo.12108)

Dreber, A., D. G. Rand, D. Fudenberg, and M. A. Nowak. (2008). Winners don't punish. Nature, 452, 348-351. (DOI 10.1038/nature06723)

Earley, R. L. (2010). Social eavesdropping and the evolution of conditional cooperation and cheating strategies. Philosophical Transactions of the Royal Society B-Biological Sciences, 365, 2675-2686. (DOI 10.1098/rstb.2010.0147) 
Fehr, E. (2004). Human behaviour: Don't lose your reputation. Nature, 432, 449-450. (DOI $10.1038 / 432449 \mathrm{a})$

Fehr, E., \& S. Gächter. (2002). Altruistic punishment in humans. Nature, 415, 137-140. (DOI $10.1038 / 415137 \mathrm{a})$

Fehrler, S., \& W. Przepiorka. (2013). Charitable giving as a signal of trustworthiness: Disentangling the signaling benefits of altruistic acts. Evolution and Human Behavior, 34, 139-145. (DOI 10.1016/j.evolhumbehav.2012.11.005)

Fu, F., C. Hauert, M. A. Nowak, and L. Wang. (2008). Reputation-based partner choice promotes cooperation in social networks. Physical Review E, 78. (DOI 10.1103/PhysRevE.78.026117)

Gardner, A., \& S. A. West. (2004). Cooperation and punishment, especially in humans. American Naturalist, 164, 753-764. (DOI 10.1086/425623)

Gintis, H., Smith, E. A., \& S. Bowles. (2001). Costly signaling and cooperation. Journal of theoretical Biology, 213, 103-119. (DOI 10.1006/jtbi.2001.2406)

Guala, F. (2012). Reciprocity: weak or strong? What punishment experiments do (and do not) demonstrate. Behavioral and Brain Sciences,35, 1-15. (DOI 10.1017/S0140525X11000069)

Hamilton, W. D. (1964). Genetical evolution of social behaviour, I \& II. Journal of theoretical Biology, 7, 1-52. (DOI 10.1016/0022-5193(64)90038-4)

Hardin, G. (1968). Tragedy of commons. Science, 162, 1243-1248.

Hauert, C. (2010). Replicator dynamics of reward \& reputation in public goods games. Journal of theoretical Biology, 267, 22-28. (DOI 10.1016/j.jtbi.2010.08.009)

Hilbe, C., \& K. Sigmund. (2010). Incentives and opportunism: from the carrot to the stick. Proceedings of the Royal Society B-Biological Sciences, 277, 2427-2433. (DOI 10.1098/rspb.2010.0065)

Hilbe, C., \& A. Traulsen. (2012). Emergence of responsible sanctions without second order free riders, antisocial punishment or spite. Scientific Reports, 2, 458. (DOI 10.1038/srep00458)

Kokko, H., R. A. Johnstone, and T. H. Clutton-Brock. (2001). The evolution of cooperative breeding through group augmentation. Proceedings of the Royal Society B-Biological Sciences 268, 187-196. (DOI 10.1098/rspb.2000.1349)

Lotem, A., Fishman, M. A., \& L. Stone. (2003). From reciprocity to unconditional altruism through signalling benefits. Proceedings of the Royal Society B-Biological Sciences, 270, 199-205. (DOI 10.1098/rspb.2002.2225)

Milinski, M., Semmann, D., \& H. J. Krambeck. (2002). Reputation helps solve the 'tragedy of the commons'. Nature, 415, 424-426. (DOI 10.1038/415424a)

Nowak, M. A., \& K. Sigmund. (1998). Evolution of indirect reciprocity by image scoring. Nature, 393, 573-577. (DOI 10.1038/31225)

Nowak, M. A., \& K. Sigmund. (2005). Evolution of indirect reciprocity. Nature, 437, 12911298. (DOI 10.1038/nature04131)

Panchanathan, K., \& R. Boyd. (2004). Indirect reciprocity can stabilize cooperation without the second-order free rider problem. Nature, 432, 499-502. (DOI 10.1038/nature02978)

Przepiorka, W., \& A. Diekmann. (2013). Individual heterogeneity and costly punishment: a volunteer's dilemma. Proceedings of the Royal Society B-Biological Sciences, 280, 20130247. (DOI 10.1098/rspb.2013.0247)

Raihani, N. J., \& R. Bshary. (2011). The evolution of punishment in n-player public goods games: a volunteer's dilemma. Evolution, 65, 2725-2728. (DOI 10.1111/j.15585646.2011.01383.x)

Raihani, N. J., A. S. Grutter, and R. Bshary. (2010). Punishers benefit from third-party punishment in fish. Science, 327, 171-171. (DOI 10.1126/science.1183068) 
Raihani, N. J., A. Thornton, and R. Bshary. (2012). Punishment and cooperation in nature. Trends in Ecology and Evolution, 27, 288-295. (DOI 10.1016/j.tree.2011.12.004)

Rankin, D. J., M. dos Santos, and C. Wedekind. (2009). The evolutionary significance of costly punishment is still to be demonstrated. Proceedings of the National Academy of Sciences USA, 106, E135. (DOI 10.1073/pnas.0911990107)

Rockenbach, B., \& M. Milinski. (2011). To qualify as a social partner, humans hide severe punishment, although their observed cooperativeness is decisive. Proceedings of the National Academy of Sciences USA, 108, 18307-18312. (DOI 10.1073/pnas.1108996108)

Sasaki, T., \& S. Uchida. (2013). The evolution of cooperation by social exclusion. Proceedings of the Royal Society B-Biological Sciences, 280, 20122498. (DOI 10.1098/rspb.2012.2498)

Sigmund, K. (2007). Punish or perish? Retaliation and collaboration among humans. Trends in Ecology and Evolution, 22, 593-600. (DOI 10.1016/j.tree.2007.06.012)

Sigmund, K. (2010). The calculus of selfishness. Princeton University Press.

Sigmund, K. (2012). Moral assessment in indirect reciprocity. Journal of theoretical Biology, 299, 25-30. (DOI 10.1016/j.jtbi.2011.03.024)

Sigmund, K., C. Hauert, and M. A. Nowak. (2001). Reward and punishment. Proceedings of the National Academy of Sciences USA, 98, 10757-10762. (DOI 10.1073/pnas.161155698)

Suzuki, S., \& E. Akiyama. (2005). Reputation and the evolution of cooperation in sizable groups. Proceedings of the Royal Society B-Biological Sciences, 272, 1373-1377. (DOI 10.1098/rspb.2005.3072)

Suzuki, S., \& E. Akiyama. (2007). Evolution of indirect reciprocity in groups of various sizes and comparison with direct reciprocity. Journal of theoretical Biology, 245, 539-552. (DOI 10.1016/j.jtbi.2006.11.002)

Sylwester, K., \& G. Roberts. (2010). Cooperators benefit through reputation-based partner choice in economic games. Biology Letters, 6, 659-662. (DOI 10.1098/rsbl.2010.0209)

Tanabe, S., Suzuki, H., \& N. Masuda. (2013). Indirect reciprocity with trinary reputations. Journal of theoretical Biology, 317, 338-347. (DOI 10.1016/j.jtbi.2012.10.031)

Tognetti, A., Berticat, C., Raymond, M., \& C. Faurie. (2014). Assortative mating based on cooperativeness and generosity. Journal of evolutionary Biology, 27, 975-981. (DOI 10.1111/jeb.12346)

Wedekind, C., \& V. A. Braithwaite. (2002). The long-term benefits of human generosity in indirect reciprocity. Current Biology, 12, 1012-1015. (DOI 10.1016/S09609822(02)00890-4)

Wedekind, C., \& M. Milinski. (2000). Cooperation through image scoring in humans. Science, 288, 850-852. (DOI 10.1126/science.288.5467.850)

West, S. A., A. S. Griffin, and A. Gardner. (2007). Evolutionary explanations for cooperation. Current Biology, 17, R661-R672. (DOI 10.1016/j.cub.2007.06.004)

Wu, J. J., B. Y. Zhang, Z. X. Zhou, Q. Q. He, X. D. Zheng, R. Cressman, and Y. Tao. (2009). Costly punishment does not always increase cooperation. Proceedings of the National Academy of Sciences USA, 106, 17448-17451. (DOI 10.1073/pnas.0905918106)

Yoeli, E., M. Hoffman, D. G. Rand, and M. A. Nowak. (2013). Powering up with indirect reciprocity in a large-scale field experiment. Proceedings of the National Academy of Sciences USA, 110, 10424-10429. (DOI 10.1073/pnas.1301210110)

Table 1

Table 1. List of symbols used in the model.

\begin{tabular}{cl}
\hline symbol & definition \\
\hline$x$ & number of partners $(x \in \in\{1,2,3, \ldots, n-1\})$
\end{tabular}




$\begin{array}{ll}y & \text { reputation of partners }(y \in \in\{-1,0,+1\}) \\ z & \text { punishment strategy }(z \in \in\{0,1\}) \\ N & \text { population size } \\ n & \text { group size } \\ c & \text { cost of helping } \\ r & \text { public good multiplication factor }(1<r<n) \\ \alpha & \text { cost of punishing } \\ \beta & \text { cost of being punished }(\alpha<\beta) \\ m & \text { average number of rounds played per individual } \\ \mu & \text { mutation rate } \\ \varepsilon & \text { error rate in reputation assessment }\end{array}$




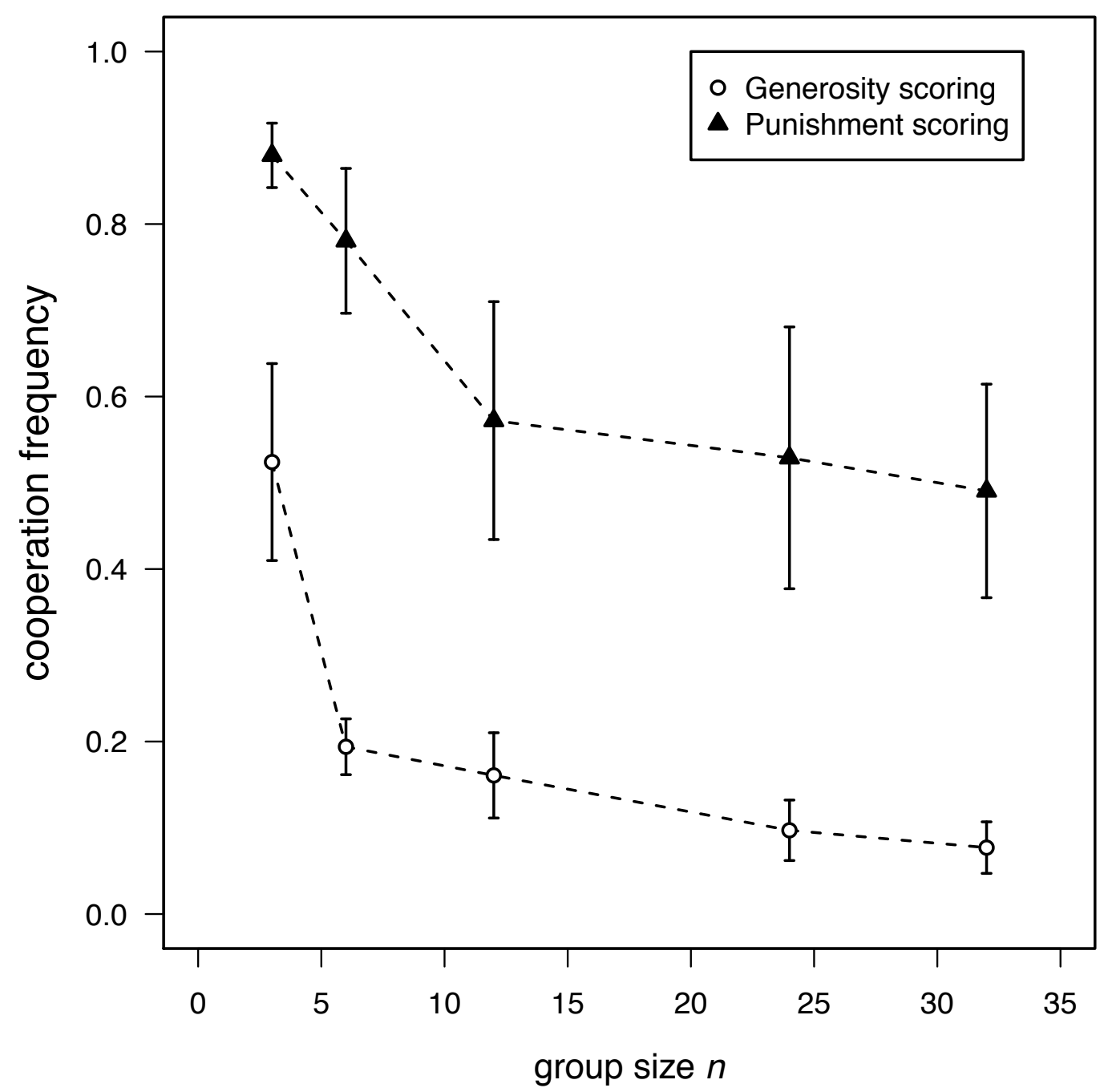

Fig. 1 Cooperation frequency (mean $\pm \mathrm{SE}$ ) dependent on group size. Cooperation rates decrease with increasing group size, and higher levels of cooperation are reached with a reputation system based on punitive (punishment scoring) rather than cooperative (generosity scoring) actions. Parameter values are: $r=0.9 n, \alpha=0.1, \beta=0.3$. 


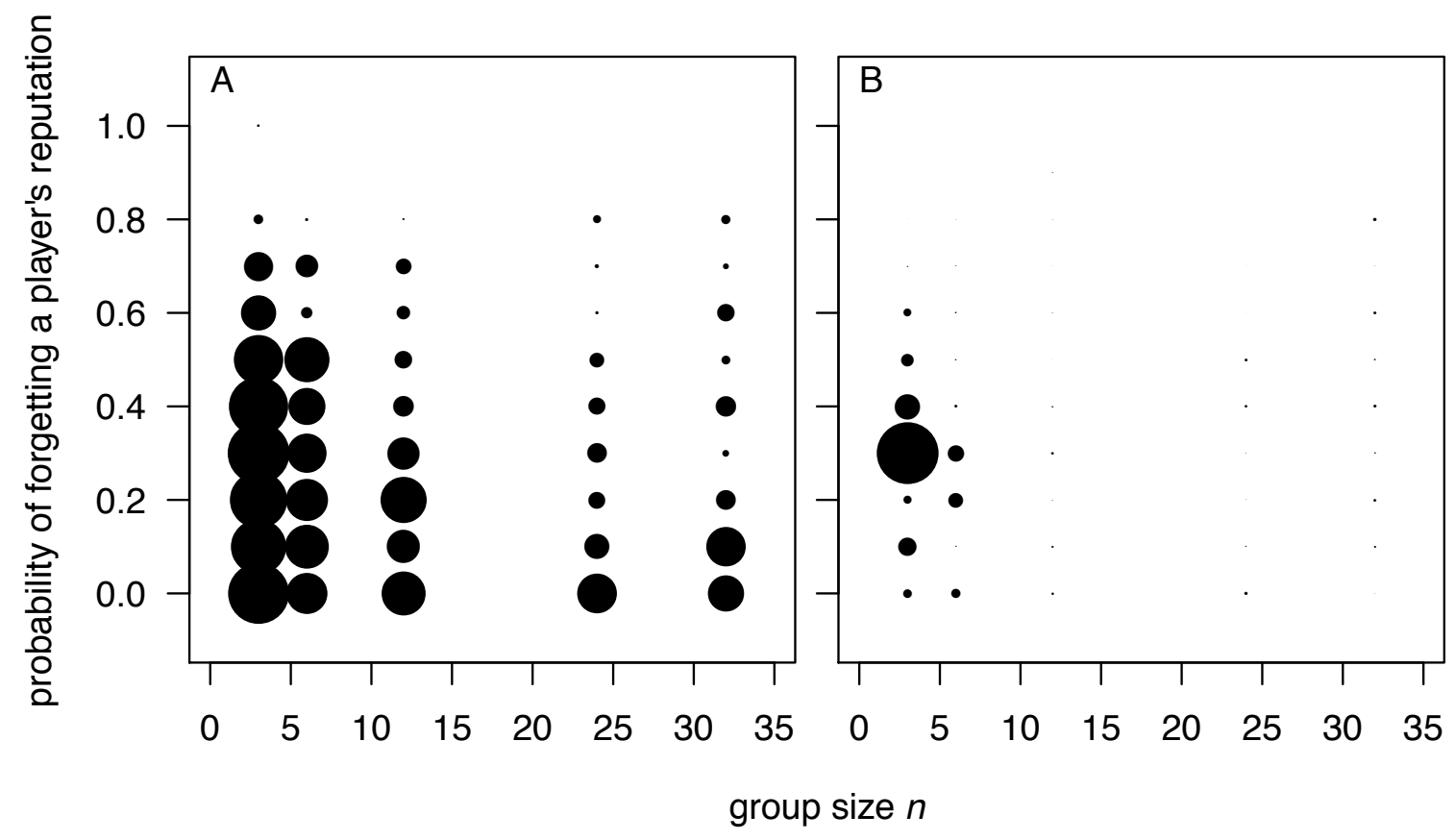

Fig.2 The effect of increasing the probability of forgetting a player's reputation $(\varepsilon)$ on cooperation frequency, indicated by the size of the circles. A reputation system based on punitive actions (A) is more robust to the inclusion of errors in reputation assessment than one based on cooperative actions (B). Parameters values are: $r=0.66 n, \alpha=0.1, \beta=0.5$. 


\section{Reputation based on punishment rather than generosity}

\section{allows for the evolution of cooperation in sizable groups}

Miguel dos Santos \& Claus Wedekind

Department of Ecology and Evolution, Biophore, University of Lausanne, 1015 Lausanne, Switzerland.

\section{Supplementary material}

Fig. A1.

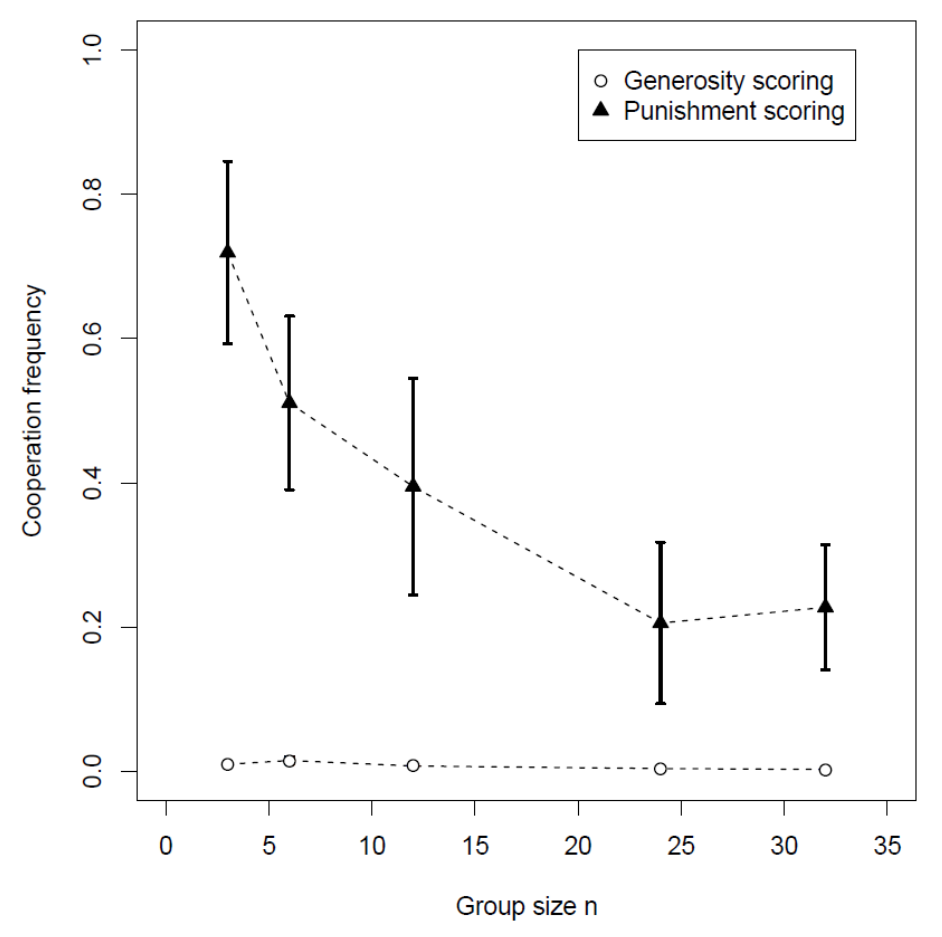

Prevalence of cooperation in function of group size (mean \pm SE). Although cooperation rates decrease with increasing group size, higher levels of cooperation are reached with a reputation system based on punitive (punishment scoring) rather than cooperative (generosity scoring) actions. Parameter values are: $r=0.66 n, \alpha=0.3, \beta=1, \varepsilon=0$. 
Fig. A2.

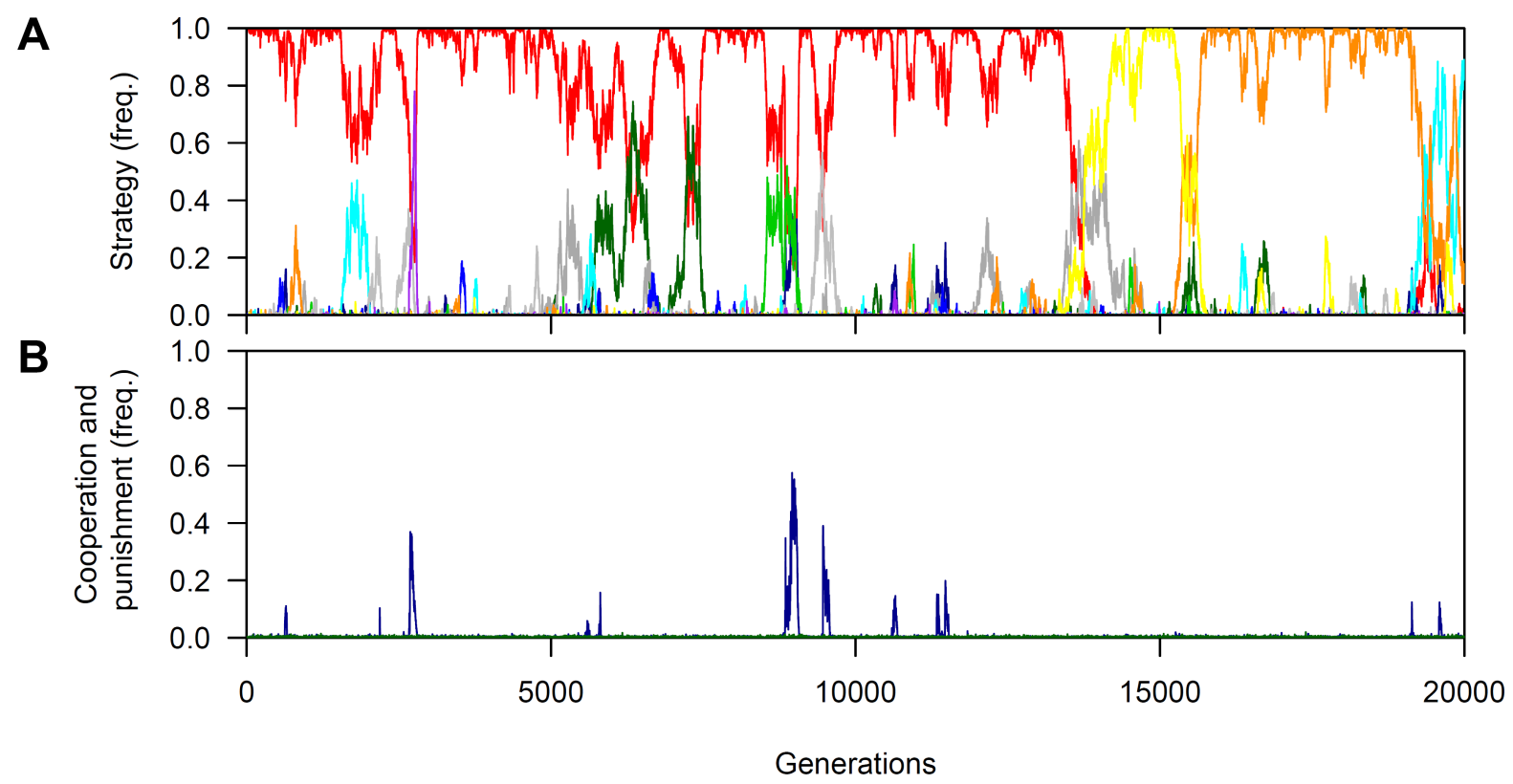

Typical simulation run with generosity scoring for a group size of 12 , with (A) frequencies of strategies and (B) frequencies of cooperation (blue) and punishment (green). Only the most frequent strategies are plotted to enhance clarity. Unconditional strategies: red: ALLD-0. Nonpunishing conditional strategies: dark blue: $\{1,1,0\}$, grey: $\{2,1,0\}$, dark green: $\{3,1,0\}$, green: $\{4,1,0\}$, dark grey: $\{5,1,0\}$, purple: $\{7,0,0\}$, blue: $\{7,1,0\}$, cyan: $\{8,1,0\}$, orange: $\{9,1,0\}$, yellow: $\{10,1,0\}$. Punishing conditional strategies: dark red: $\{3,0,1\}$, light blue: $\{4,0,1\}$. Parameters values are: $r=0.66 n, \alpha=0.1, \beta=0.3, \varepsilon=0$. 
Fig. A3.

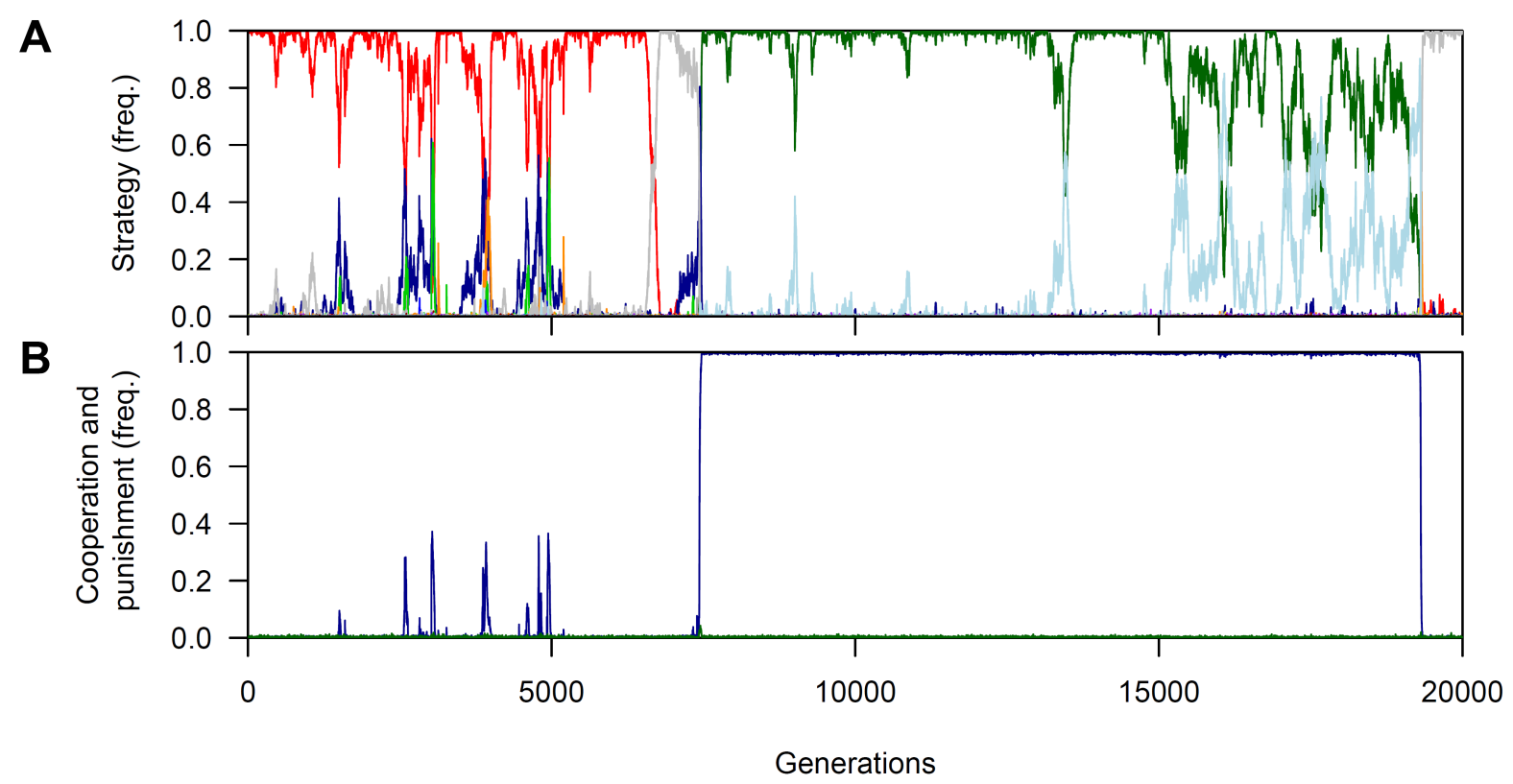

Typical simulation run under generosity scoring for a group size of 3, with (A) frequencies of strategies and (B) frequencies of cooperation (blue) and punishment (green). Periods of cooperative states can sometimes be stabilized with punishment, but usually collapse as are able to invade. For clarity, only the most frequent strategies are plotted. Unconditional strategies: red: ALLD-0, light blue: ALLC-0, dark green: ALLC-1, dark red: ALLD-1. Non-punishing conditional strategies: dark grey: $\{1,-1,0\}$, green: $\{1,0,0\}$, dark blue: $\{1,1,0\}$, blue: $\{2,-1,0\}$, cyan: $\{2,-1,1\}$, orange: $\{2,0,0\}$, yellow: $\{2,0,1\}$, grey: $\{2,1,0\}$, purple: $\{2,1,1\}$. Parameters values are: $r=$ $0.66 n, \alpha=0.1, \beta=1, \varepsilon=0$. 
Fig. A4

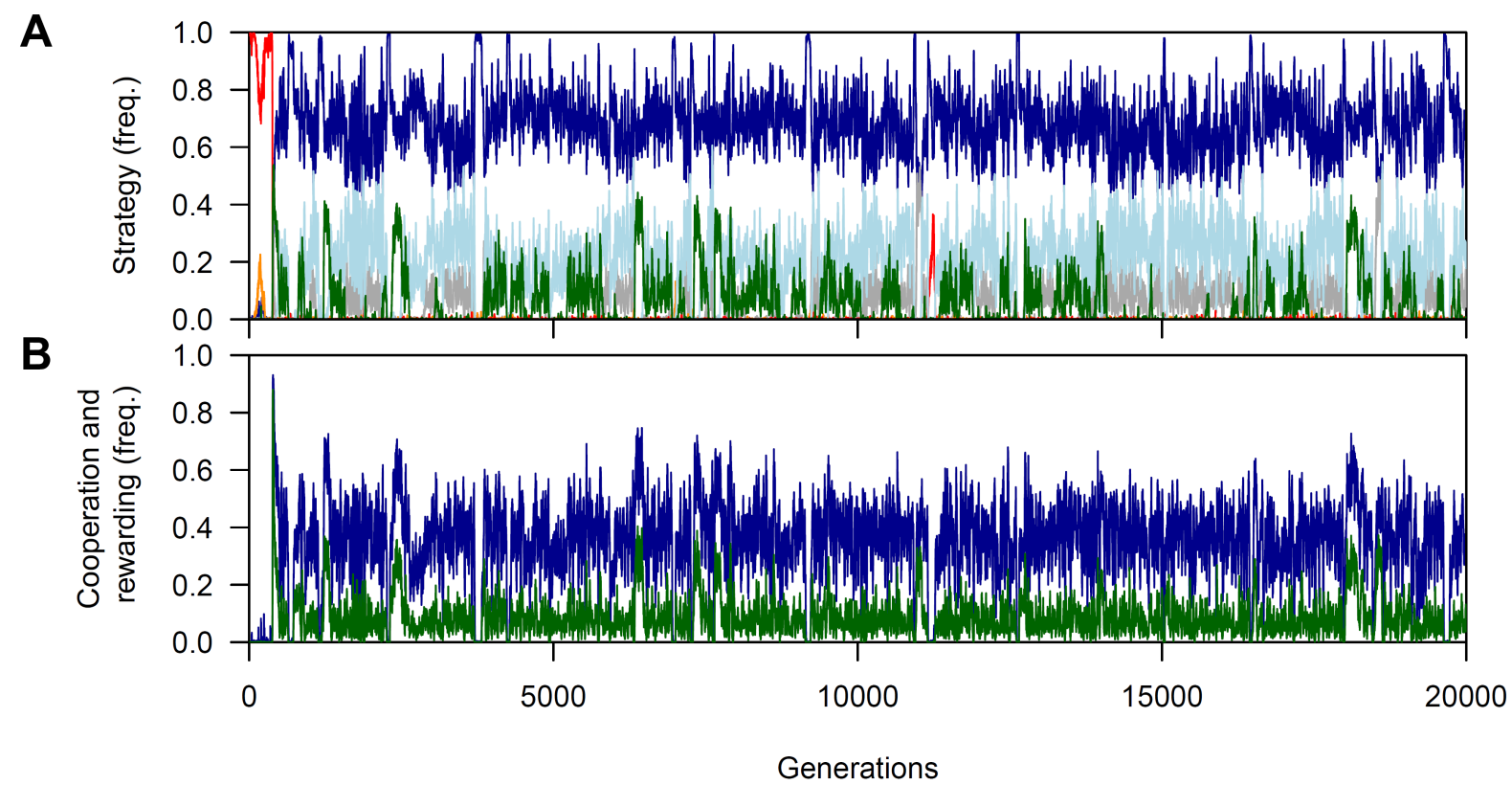

Typical simulation run with reward scoring for a group size of 3, with (A) frequencies of strategies and (B) frequencies of cooperation (blue) and rewarding (green). Cooperation quickly appears in the simulations but fails to be stabilized, resulting in a mixture of rewarding and nonrewarding strategies. For clarity, only the most frequent strategies are plotted. Unconditional strategies: red: ALLD-0, light blue: ALLC-0, dark green: ALLC-1. Non-rewarding conditional strategies: dark blue: $\{1,1,0\}$, orange: $\{2,-1,0\}$. Rewarding conditional strategies: dark grey: $\{1,1,1\}$. Parameters values are: $r=0.9 n, \alpha=0.5, \beta=1.5, \varepsilon=0$. 
Fig. A5

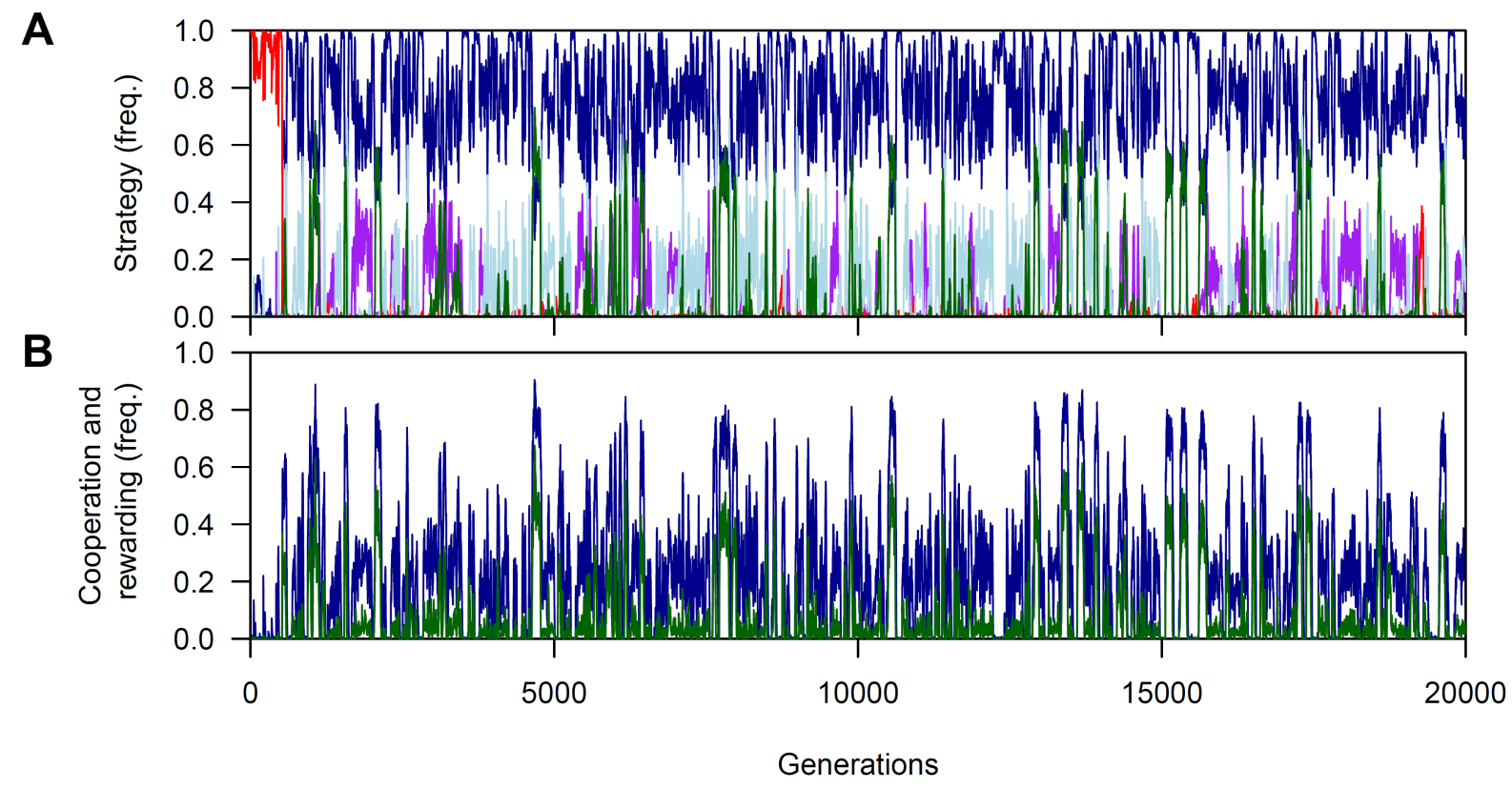

Typical simulation run with reward scoring for a group size of 3 with errors in reputation assessment, with (A) frequencies of strategies and (B) frequencies of cooperation (blue) and rewarding (green). For clarity, only the most frequent strategies are plotted. Unconditional strategies: red: ALLD-0, light blue: ALLC-0, dark green: ALLC-1. Non-rewarding conditional strategies: dark blue: $\{1,1,0\}$, purple: $\{1,0,0\}$. Rewarding conditional strategies: dark grey: $\{1,1,1\}$. Parameters values are: $r=0.9 n, \alpha=0.5, \beta=1.5, \varepsilon=0.3$. 
Fig. A6.

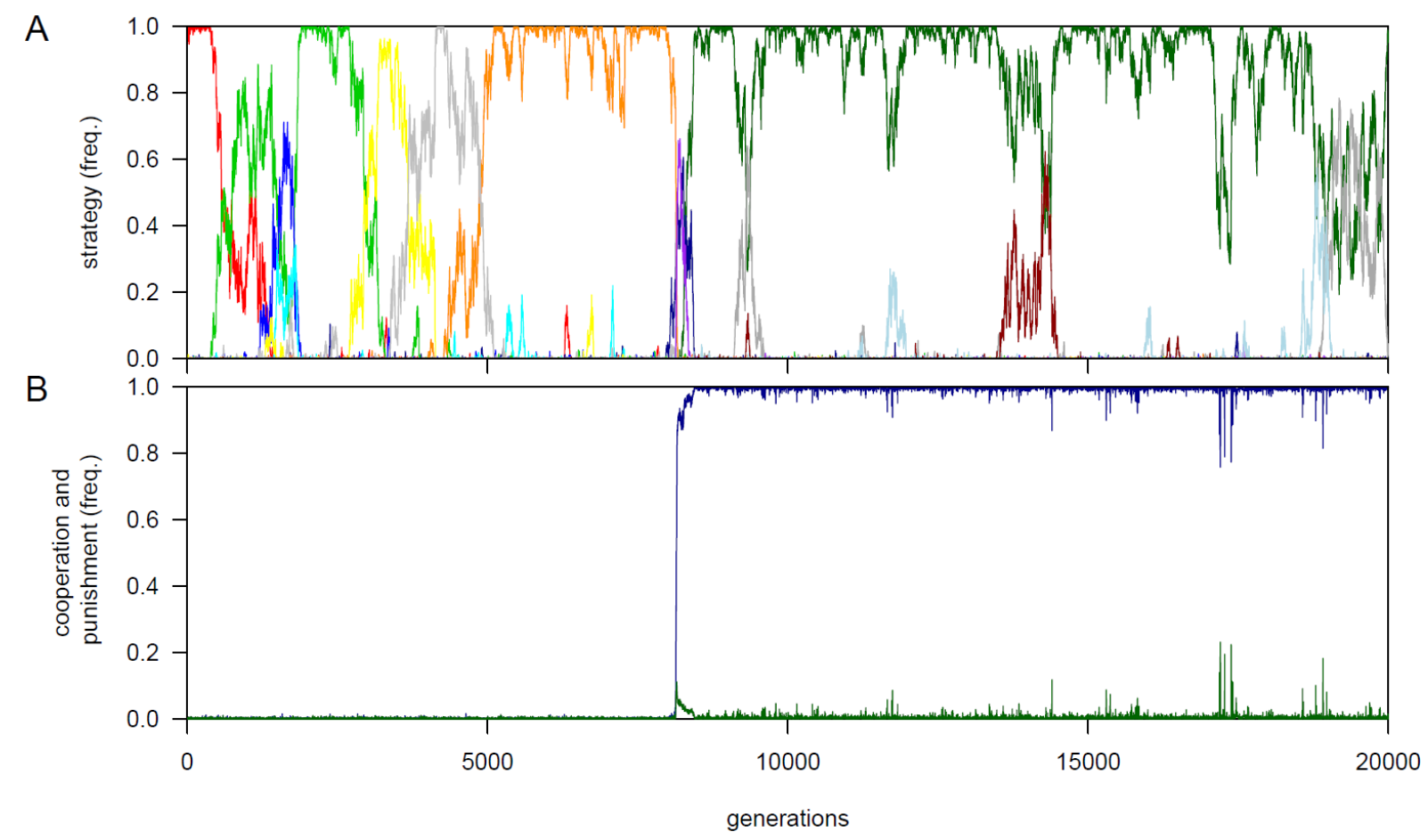

A typical simulation run for $\mathrm{n}=12$ with a reputation system based on punitive actions (punishment scoring), with (A) frequencies of strategies and (B) frequencies of cooperation (blue) and punishment (green). Only the most frequent strategies are plotted. Red: ALLD-0, dark green: ALLC-1. Non-punishing conditional strategies: green $\{4,1,0\}$, dark blue $\{1,1,0\}$, blue $\{7,1,0\}$, cyan $\{8,1,0\}$, orange $\{9,1,0\}$, yellow $\{10,1,0\}$, grey $\{11,1,0\}$. Punishing conditional strategies: dark grey $\{1,0,1\}$, blue $\{1,1,1\}$, purple $\{2,1,1\}$, dark red $\{3,0,1\}$, light blue $\{4,0,1\}$. Parameters values are: $r=0.66 n, \alpha=0.1, \beta=1$. 
Fig. A7.

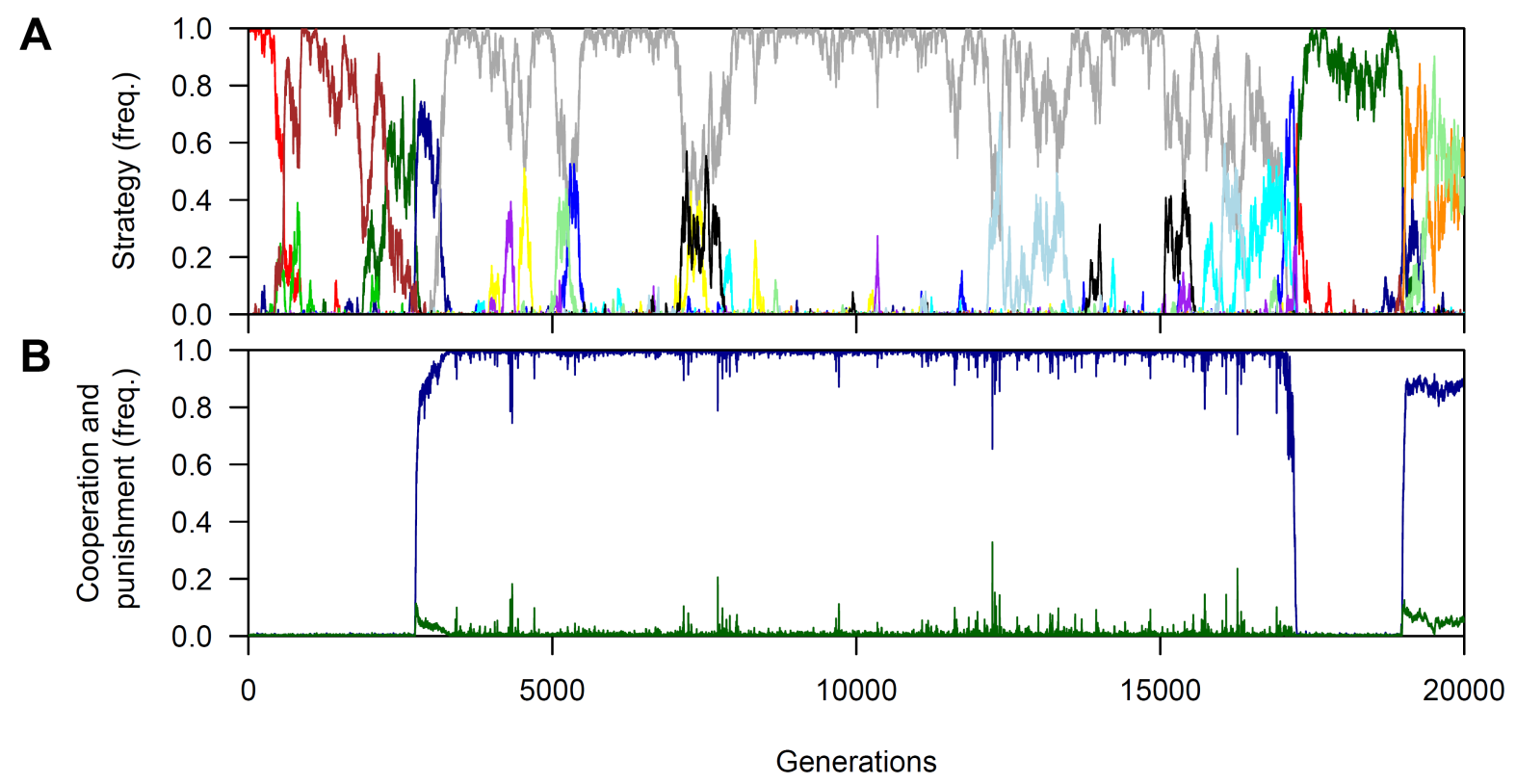

A typical simulation run under punishment scoring for a group size of 12 , with (A) frequencies of strategies and (B) frequencies of cooperation (blue) and punishment (green). Periods of cooperation could intermittently collapse as discriminatory strategies can be invaded by punishing unconditional cooperators. The latter can then be invaded by non-punishing unconditional cooperators who can in turn be invaded by non-punishing defectors. For clarity, only the most frequent strategies are plotted. Unconditional strategies: red: ALLD-0, light green: ALLC-0. Non-punishing conditional strategies: blue: $\{1,0,0\}$, dark blue: $\{1,1,0\}$, cyan: $\{2,0,0\}$, dark green: $\{3,1,0\}$, green: $\{4,1,0\}$, purple: $\{5,0,0\}$, brown: $\{5,1,0\}$. Punishing conditional strategies: dark grey: $\{1,0,1\}$ yellow: $\{2,0,1\}$, black: $\{3,0,1\}$, orange: $\{3,1,1\}$, light blue: $\{4,0,1\}$, grey: $\{5,1,1\}$. Parameters values are: $r=0.66 n, \alpha=0.1, \beta=0.3, \varepsilon=0$. 
Fig. A8.

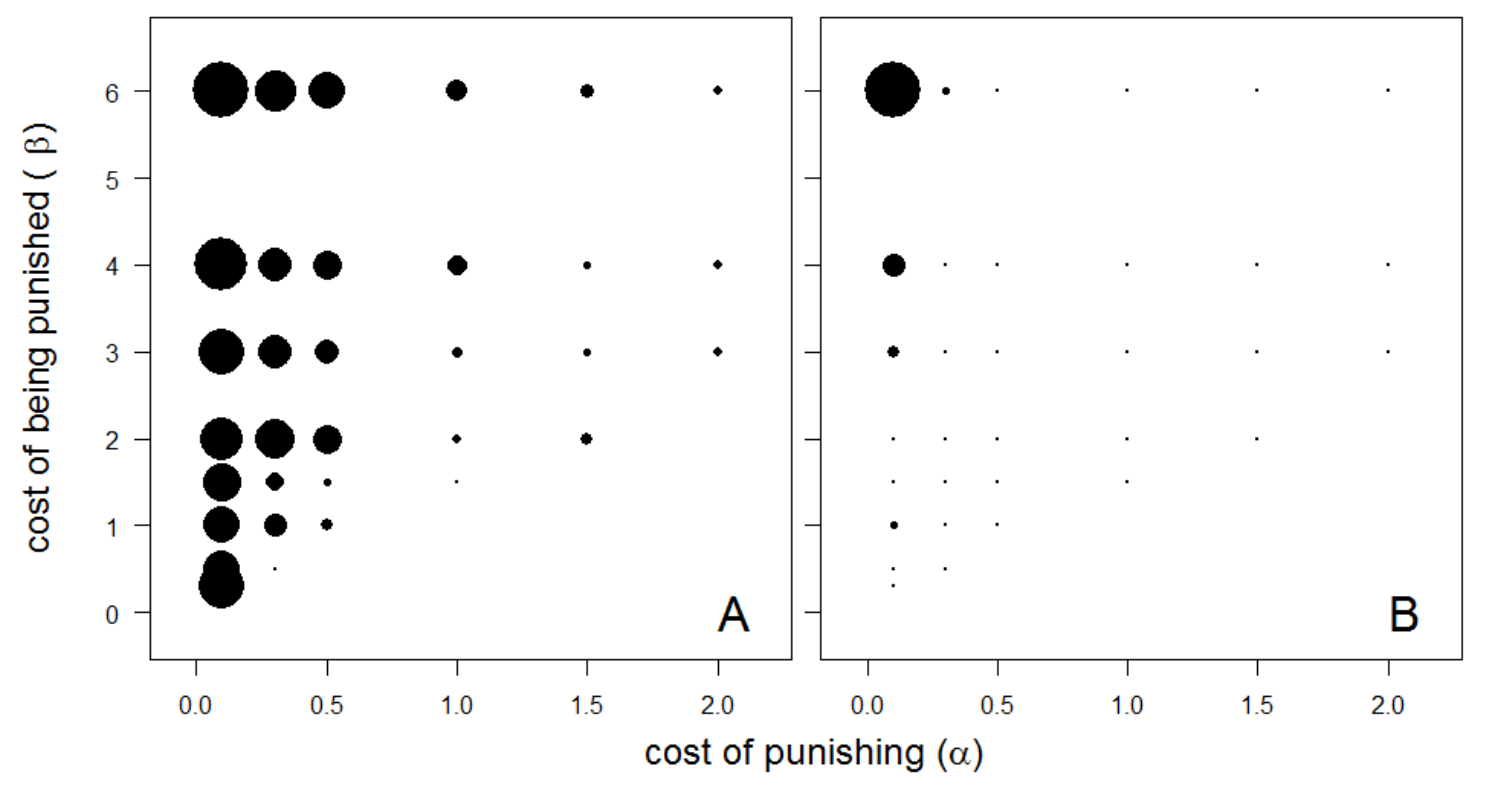

Cooperation frequency for different punishment costs $(\alpha)$ and impacts $(\beta)$ for a group size of 12 with punishment scoring (A) and generosity scoring (B). With punishment scoring, higher cooperation rates are reached for lower punishment costs and impacts. Circle size represents cooperation frequency. Parameters values are: $r=0.66 n, \varepsilon=0$. 
Fig. A9

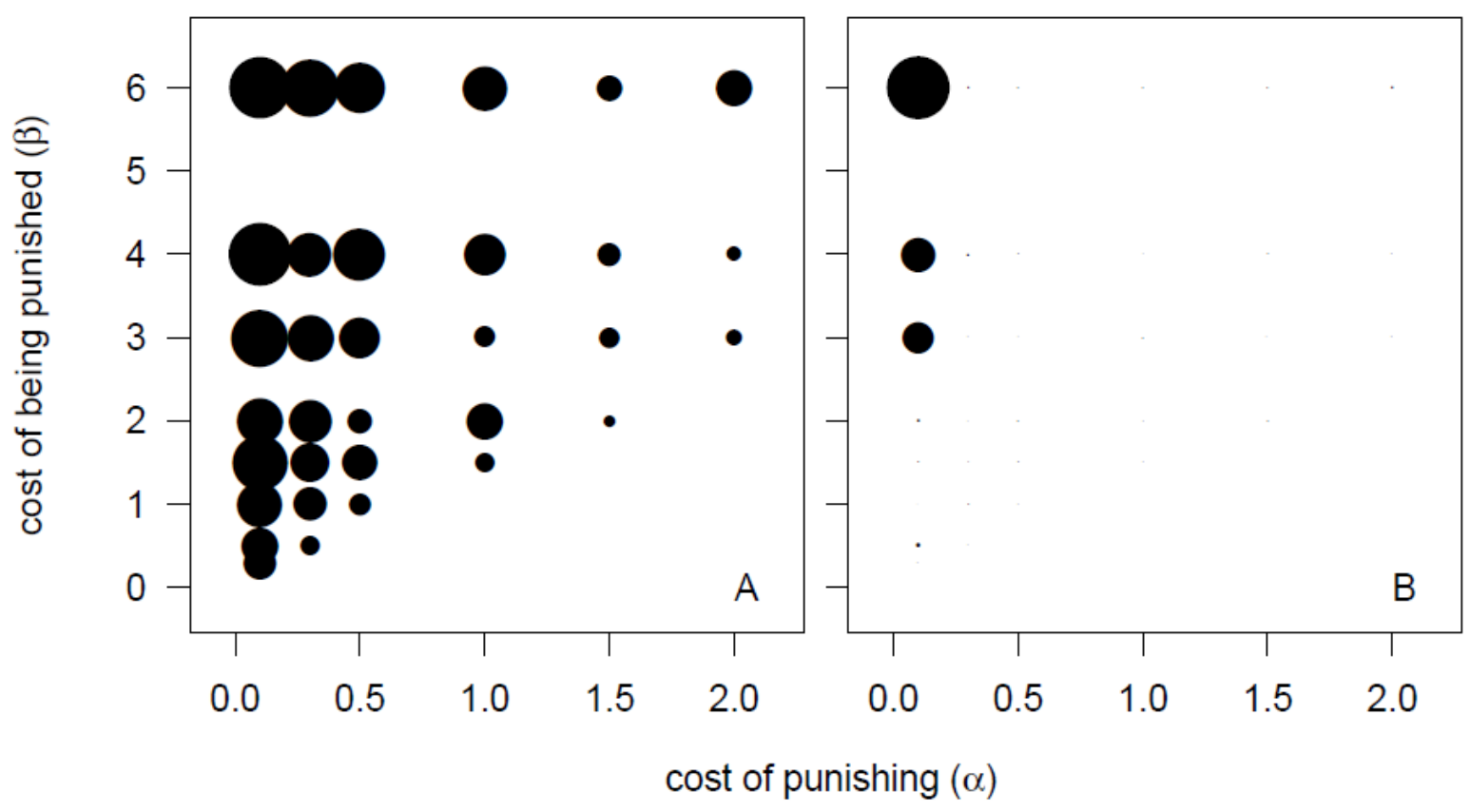

Cooperation frequency for different costs of punishing $(\alpha)$ and being punished $(\beta)$ with punishment scoring (A) and generosity scoring (B). Circle size represents cooperation frequency. Parameters values are: $n=6, r=0.66 n, \varepsilon=0$. 
Fig. A10.

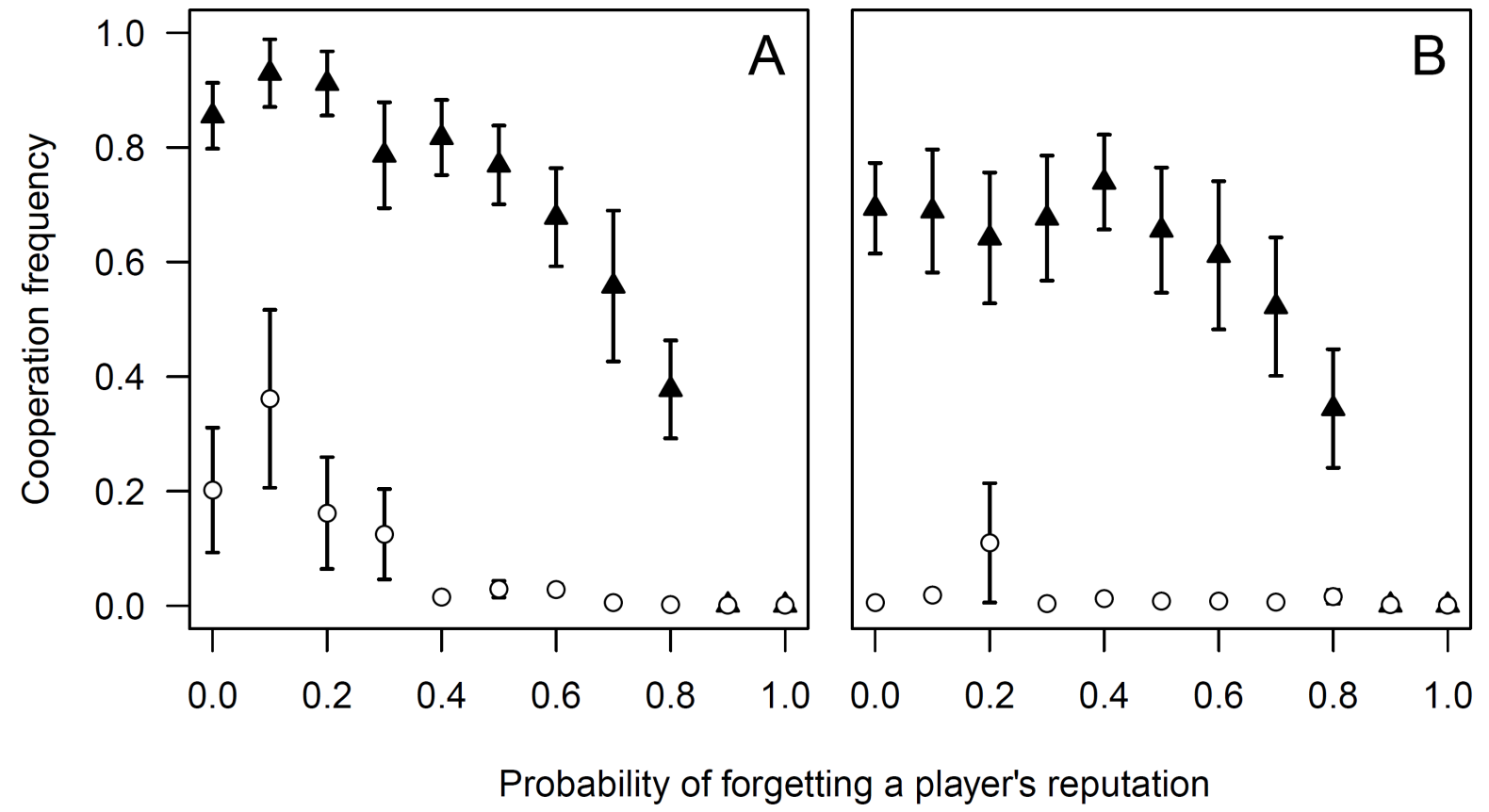

Effect of increasing the probability of forgetting a player's reputation with punishment scoring (triangles) and generosity scoring (circles) for a group size of 3 (A) and 6 (B). Parameter values are: $r=0.66 n, \alpha=0.1, \beta=1$. 
Fig. A11.

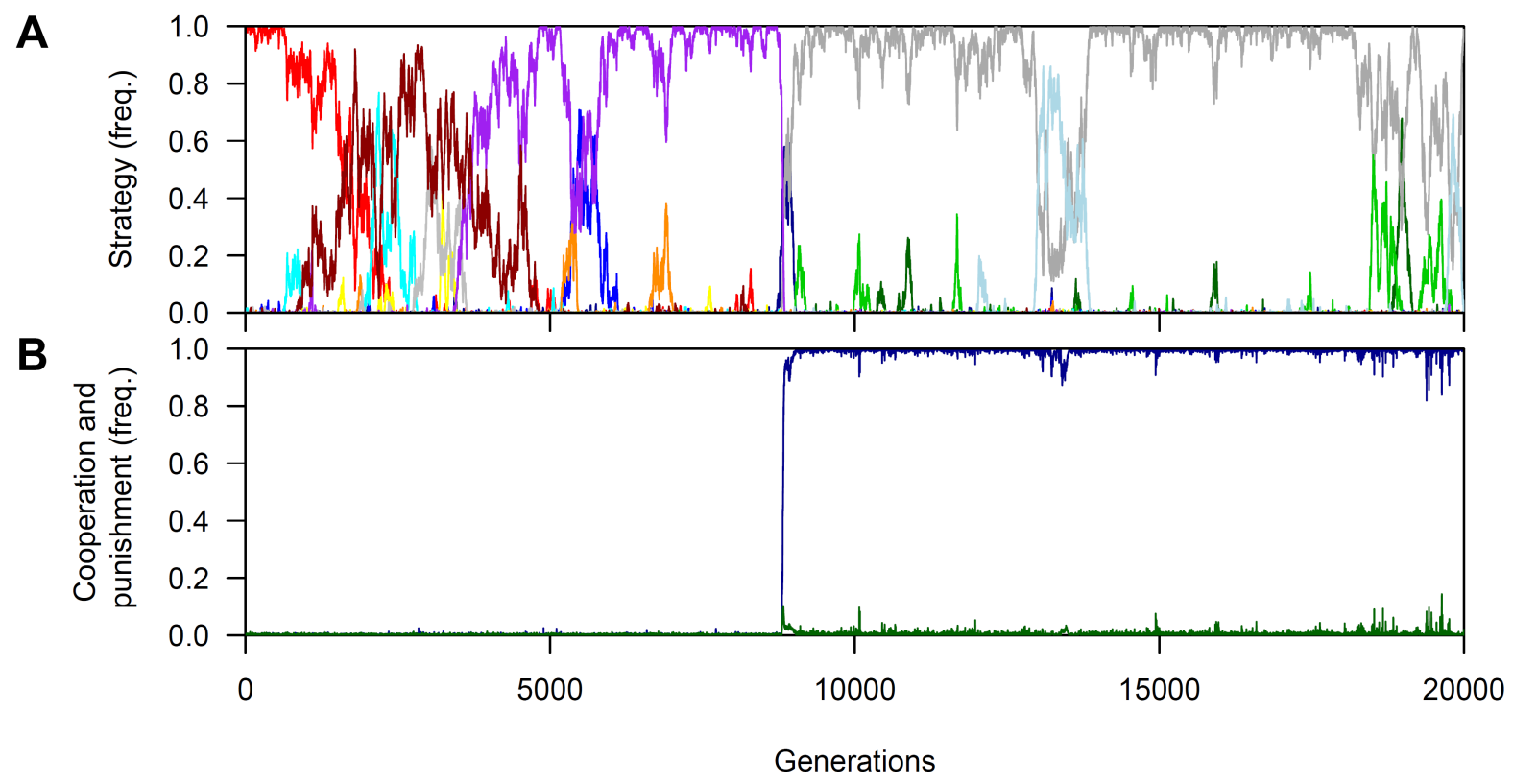

Typical simulation run with punishment scoring for a group size of 12 , with (A) frequencies of strategies and (B) frequencies of cooperation (blue) and punishment (green). Even though the probability of forgetting a player's reputation is $\varepsilon=0.3$, cooperation can still emerge. For clarity, only the most frequent strategies are plotted. Unconditional strategies: red: ALLD-0, light blue: ALLC-0, dark green: ALLC-1. Non-punishing conditional strategies: dark blue: $\{1,1,0\}$, , cyan: $\{3,1,0\}$, purple: $\{5,1,0\}$, dark red: $\{6,1,0\}$, blue: $\{7,1,0\}$, orange: $\{9,1,0\}$, yellow: $\{10,1,0\}$, grey: $\{11,1,0\}$. Punishing conditional strategies: dark grey: $\{1,0,1\}$, green: $\{6,0,1\}$. Parameters values are: $r=0.66 n, \alpha=0.1, \beta=0.3$. 
Fig. A12

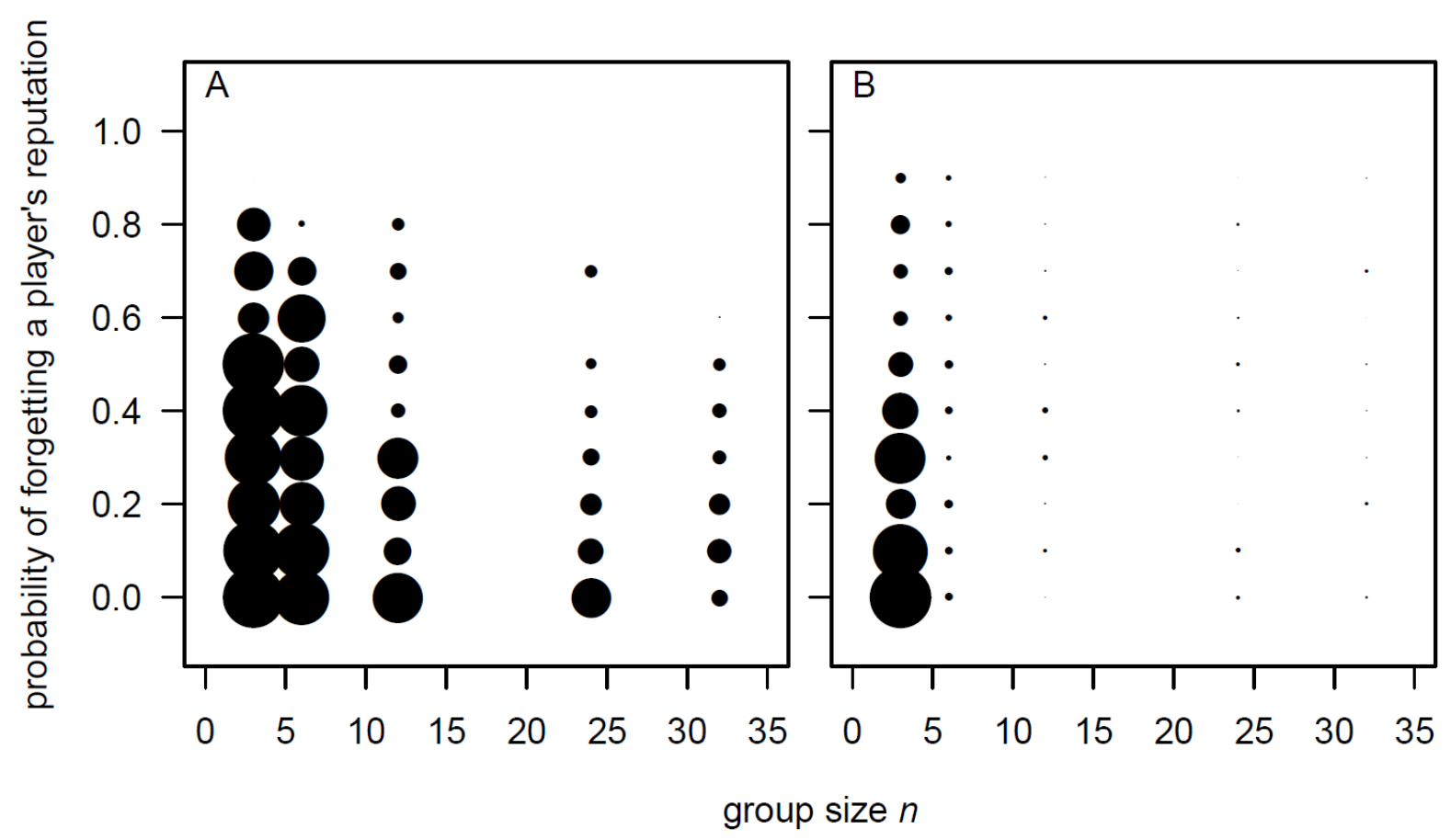

The effect of increasing the probability of forgetting a player's reputation $(\varepsilon)$ on cooperation frequency, indicated by the size of the circles. A reputation system based on punitive actions (A) is more robust to the inclusion of errors in reputation assessment than one based on cooperative actions (B). Parameters values are: $N=2500, r=0.9 n, \alpha=0.1, \beta=0.3, \mu=$ 0.0004 . 
Fig. A13

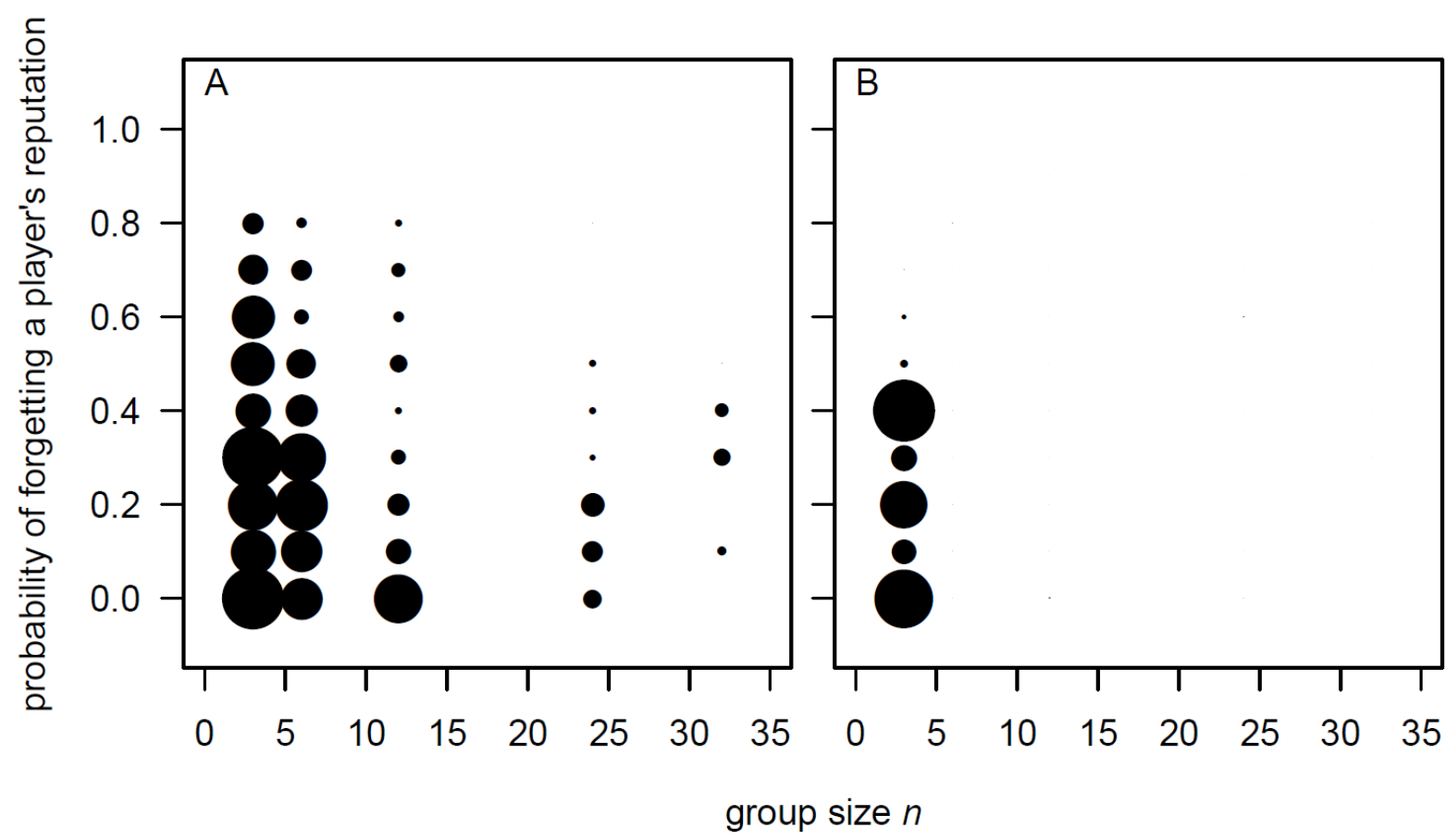

The effect of increasing the probability of forgetting a player's reputation $(\varepsilon)$ on cooperation frequency, indicated by the size of the circles. A reputation system based on punitive actions (A) is more robust to the inclusion of errors in reputation assessment than one based on cooperative actions (B). Parameters values are: $N=2500, r=0.66 n, \alpha=0.1, \beta=0.5, \mu=$ 0.0004 . 
Fig. A14

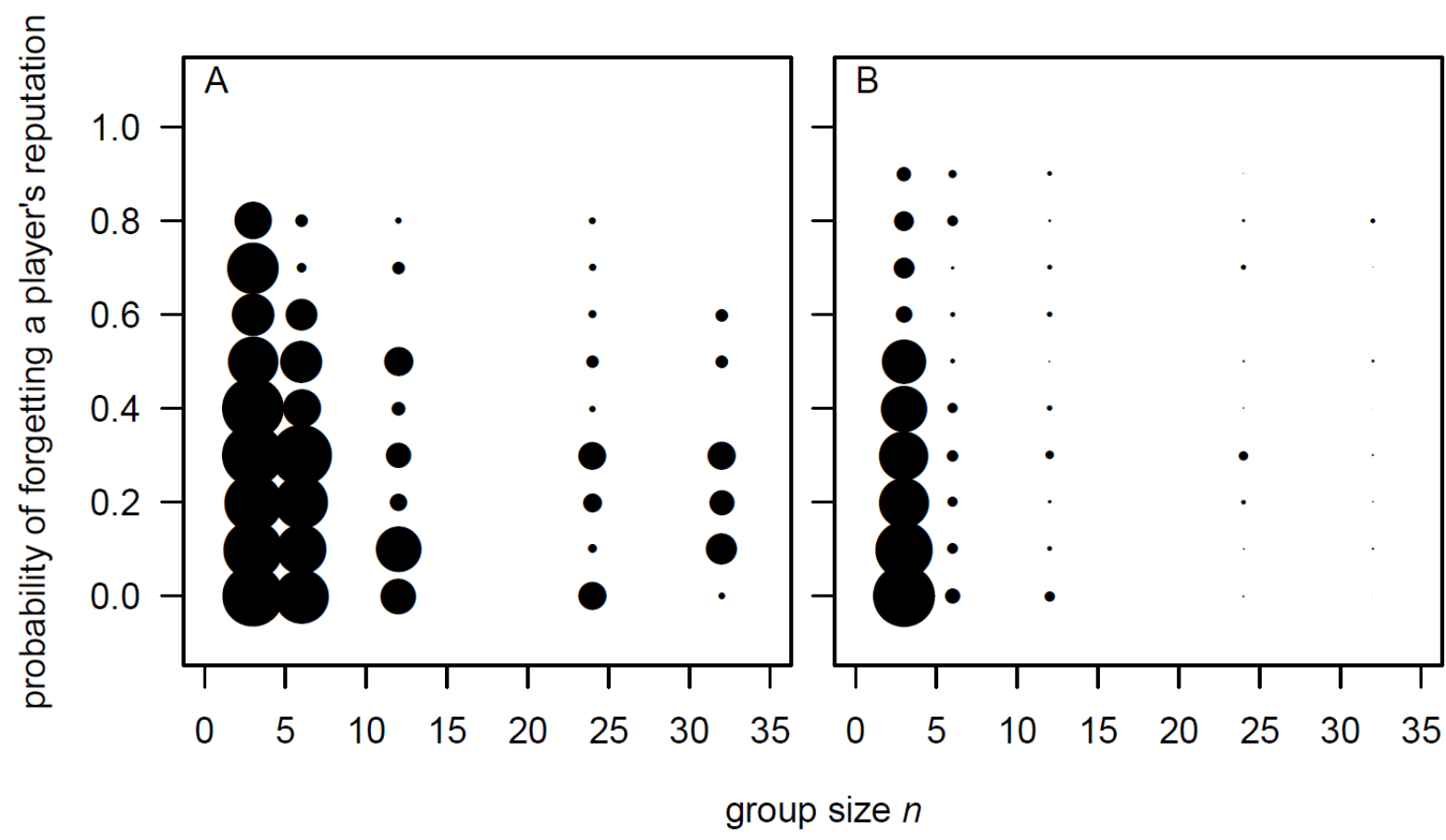

The effect of increasing the probability of forgetting a player's reputation $(\varepsilon)$ on cooperation frequency, indicated by the size of the circles. A reputation system based on punitive actions (A) is more robust to the inclusion of errors in reputation assessment than one based on cooperative actions (B). Parameters values are: $N=5000, r=0.9 n, \alpha=0.1, \beta=0.3, \mu=$ 0.0002 . 
Fig. A15

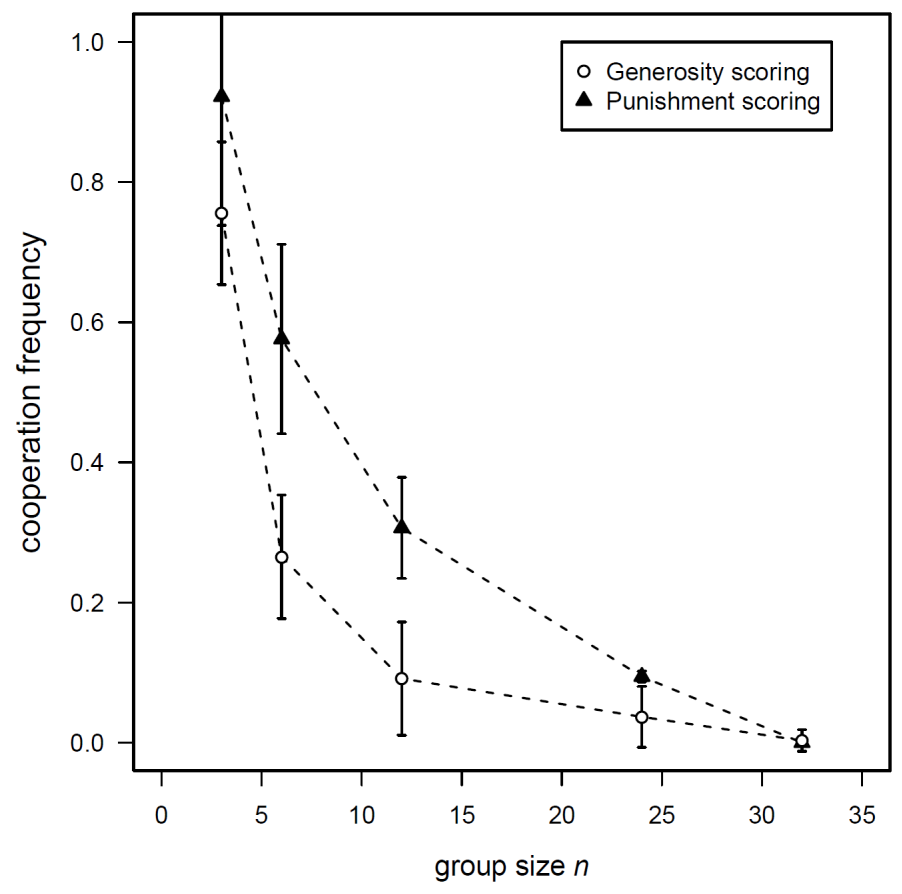

Prevalence of cooperation in function of group size (mean \pm SE). Although cooperation rates decrease with increasing group size, higher levels of cooperation are reached with a reputation system based on punitive (punishment scoring) rather than cooperative (generosity scoring) actions. Parameter values are: $N=10^{4}, r=0.9 n, \alpha=0.1, \beta=0.1, \varepsilon=0, \mu=10^{-4}$. 